\title{
SLR TECHNIQUE USED FOR DESCRIPTION OF THE EARTH ELASTICITY
}

\author{
Miłosława Rutkowska, Marcin Jagoda \\ Technical University of Koszalin, Śniadeckich 2, 75-453 Koszalin, Poland, \\ e-mail: marcin.jagoda@wilsig.tu.koszalin.pl,milena@cbk.waw.pl
}

\begin{abstract}
We present estimated values for the global elastic parameters $\left(h_{2}, l_{2}\right)$ derived from the analysis of Satellite Laser Ranging (SLR) data. We analyse SLR data for LAGEOS 1 and LAGEOS 2 and for two low satellites, STARLETTE and STELLA, collected over a period of 2.5 years, from January 12005 to July 1 2007, from 18 globally distributed ground stations. The adjusted final values $\left(h_{2}, l_{2}\right)$ for all satellites are compared. A major discrepancy between the two solutions was only found for the Shida number $l_{2}$. Computations were performed using GEODYN II NASA/GSFC software.

The following analysis is the continuation of our research published in Jagoda and Rutkowska (2013); that analysis was conducted separately for the two low satellites, STELLA and STARLETTE (separately for STELLA and separately for STARLETTE) whereas in this study we present the results of determining $h_{2}$ and $l_{2}$ parameters obtained from the joint observation of the STELLA and STARLETTE satellites (STARLETTE + STELLA) and joint observation of high satellites: LAGEOS 1 and LAGEOS 2 (LAGEOS 1 + LAGEOS 2). The combination of the observation aims at an increased stability of the estimates and reduced errors of the means of the parameters being determined.
\end{abstract}

Key words: SLR data, Love and Shida numbers, Earth elasticity

\section{GLOBAL GRAVITY FIELD AND LOVE NUMBERS THEORY}

The gravitational and tidal potential outside the Earth at point $P(r, \varphi, \lambda)$ in a rotating frame can be expanded into a series of spherical harmonics (Eanes et al. 1983).

$$
V_{\text {Grav }}=\frac{G M_{\text {Earth }}}{r}\left\{1+\sum_{n=2}^{\infty} \sum_{m=0}^{n}\left(\frac{a_{e}}{r}\right)^{n} P_{n m}(\sin \varphi)\left(\left(C_{n m}+\Delta C_{n m}\right) \cos m \lambda+\left(S_{n m}+\Delta S_{n m}\right) \sin m \lambda\right)\right\}
$$

where:

$$
\begin{array}{ll}
G M_{\text {Earth }} & \text { - gravitational parameter for the Earth, } \\
a_{e} & \text { - radius of the Earth, } \\
P_{n m}(\sin \varphi) & \text { - associated Legendre functions, } \\
C_{n m}, S_{n m} & \text { - unnormalized geopotential coefficients, } \\
\Delta C_{n m}, \Delta S_{n m} & \text { - unnormalized corrections of geopotential coefficients, } \\
n, m & \text { - index of degree and order of the spherical harmonics. }
\end{array}
$$


The tide force caused by the Sun and the Moon and other celestial bodies tries to deform the equipotential surfaces of the Earth's gravity field, elongating their shape in the direction of the resultant force exerted by the configuration of all celestial bodies. The tides of the viscoelastic Earth change the gravitational potential of the Earth and thus cause displacement of the Earth's mass and additional accelerations acting on the satellite leading to additional perturbations of the orbit. The solid tide induced free space potential is most easily modelled as variations in the normalized correction of geopotential coefficients $\Delta \bar{C}_{n m}, \Delta \bar{S}_{n m}$ (Eanes et al. 1983), where $k_{n m}$ is the potential Love number of degree and order $(n, m)$.

$$
\Delta \bar{C}_{n m}-i \Delta \bar{S}_{n m}=\frac{k_{n m}}{\sqrt{2 n+1}} \sum_{j=2}^{3} \frac{G M_{j}}{G M_{\text {Earth }}}\left(\frac{R_{\text {Earth }}}{R_{j}}\right)^{n+1} P_{n m}\left(\sin \Phi_{j}\right) e^{-i m \lambda_{j}}
$$

where:

$R_{\text {Earth }} \quad$ - equatorial radius of the Earth,

$R_{j} \quad$ - distance from geocenter to the Moon and the Sun,

$\Phi_{j}, \lambda_{j} \quad$ - geocentric latitude and east longitude (from Greenwich) of the Moon and the Sun.

The unnormalized coefficients $C_{n m}, S_{n m}$ given in expression (1) can be computed very simply by the summation of the coefficients of the gravitational potential of the Earth for selected model and unnormalized corrections of geopotential coefficients $\Delta C_{n m}, \Delta S_{n m}$.

The normalized geopotential coefficients are related to the unnormalized coefficients by (Petit and Luzum 2010):

$$
C_{n m}=N_{n m} \bar{C}_{n m}, \quad S_{n m}=N_{n m} \bar{S}_{n m}
$$

where $N_{n m}$ is normalization coefficient:

$$
\delta_{\text {om }}=\left\{\begin{array}{ll}
1 & m=0 \\
0 & m \neq 0
\end{array} \quad \sqrt{\frac{(n-m) !(2 n+1)\left(2-\delta_{o m}\right)}{(n+m) !}}\right.
$$

Corrrespondingly, the normalized correction of geopotential coefficients are related to the unnormalized by:

$$
\Delta C_{n m}=N_{n m} \Delta \bar{C}_{n m}, \quad \Delta S_{n m}=N_{n m} \Delta \bar{S}_{n m}
$$

The Earth is a deformed visco-elastic body. The point on the Earth surface's before and after deformation defines the model of elastic deformability and can be represented by a combination of Love numbers. The Love number $h$ represents the ratio of the radial displacement of the element of mass of the elastic Earth to the corresponding displacement for a liquid Earth. The Love number $h$ is equal to 0 for the hypothetical solid Earth but equal to 1 for the liquid Earth. For the real elastic body, $0<h<1$. The much smaller Shida number $l$ represents the ratio of the transverse displacement of the element of mass of the crust to the displacement for the hypothetical liquid Earth. For the elastic Earth, $0<l<1$.

The Earth's shape changes as a response to variations of the tide generating potential. The tidal displacement of the Earth mass results in oscillations of station positions which changes the distance between the laser system and the satellite position for epoch $t_{i}$. This has a great influence on the observation equation (7) and solution accuracy. 
In approximation, when nominal values $h_{2}$ and $l_{2}$ are used combined to all degree 2 tidal terms, vector displacements in terms of the station position are given by following formulae (Diamante and Williamson 1972):

$$
\Delta r=\sum_{j=2}^{3}\left[\frac{G M_{j} R_{\text {Earth }}^{4}}{G M_{\text {Earth }} R_{j}^{3}}\right]\left\{h_{2} \hat{r}\left(\frac{3}{2}\left(\hat{R}_{j} \hat{r}\right)^{2}-\frac{1}{2}\right)+3 l_{2}\left(\hat{R}_{j} \hat{r}\right)\left[\hat{R}_{j}-\left(\hat{R}_{j} \hat{r}\right) \hat{r}\right]\right\}
$$

where:

$$
\begin{array}{ll}
G M_{j} & \text { - gravitational parameter for the Moon }(\mathrm{j}=2) \text { or the } \operatorname{Sun}(\mathrm{j}=3), \\
\hat{R}_{j} & \text { - the unit vector from the geocenter to the Moon or Sun, } \\
\hat{r}, r & \text { - the unit vector from the geocenter to the station and the magnitude of that } \\
& \text { vector, } \\
h_{2} & \text { - nominal second degree Love number, } \\
l_{2} & \text { - nominal second degree Shida number. }
\end{array}
$$

The second degree Love and Shida numbers are determined using Satellite Laser Ranging (SLR) data. From a given set of laser observations, a set of satellite orbit parameters and geophysical parameters are calculated using the least squares method. The least squares solution selects the estimated unknowns that minimize the sum of the squares of the calculated observation residuals.

This method permits the geodetic and geophysical parameters of the Earth to be determined from the LAGEOS 1, LAGEOS 2, STARLETTE and STELLA orbital arcs given by expression (1) and tide correction of station position given by expression (6). The computations are performed in two parts. In the first part, six parameters determining the position and velocity of the satellite at initial epoch, atmospheric drag coefficients $C_{D}$ for low satellites, radiation pressure coefficients $C_{R}$ must be found for each orbital arc low and high satellites. In the next part, unknowns $\left(h_{2}, l_{2}\right)$ are added: and all unknowns are adjusted in a common solution.

Knowledge of the partial derivatives described in Rutkowska and Jagoda (2010) allows $\left(h_{2}, l_{2}\right)$ to be adjusted using observation equation $(7)$ in an iterative process.

$v_{\rho}=\sum_{j=1}^{n} \frac{\partial \rho}{\partial \varepsilon} \Delta \varepsilon+\frac{\partial \rho}{\partial h_{2}} \Delta h_{2}+\frac{\partial \rho}{\partial l_{2}} \Delta l_{2}+(O-C)$

where:

$\Delta \varepsilon \quad$ - corrections for satellite position, velocity and other unknowns connected with the satellite orbit (coefficient of the solar radiation pressure, atmospheric drag, range biases, accelerations) and the station positions, velocities, where $n$ is the number of estimated unknowns,

$\Delta h_{2} \quad$ - correction for the Love number $h_{2}$,

$\Delta l_{2} \quad$ - correction for the Shida number $l_{2}$,

$v_{\rho} \quad$ - correction for observation,

$(O-C) \quad$ - SLR observation minus computed distance from station to satellite,

$\rho \quad-$ SLR distance measurement. 


\section{SATELLITES DESCRIPTION AND MEASUREMENT DATA}

Modern SLR systems have a measurement accuracy of about $1 \mathrm{~cm}$ or better, which allows the technique to contribute to a broad range of applications in geodesy and geodynamics. In this study, we carry out a sequential analysis of Love numbers estimation using two high SLR satellites: LAGEOS $1 \mathrm{~h}=5860 \mathrm{~km}$, LAGEOS $2 \mathrm{~h}=5620 \mathrm{~km}$ and two low SLR satellites: STARLETTE $\mathrm{h}=810 \mathrm{~km}$ and STELLA $\mathrm{h}=800 \mathrm{~km}$. Also other space geodetic techniques are capable of determining Love and Shida numbers, for example: Gubanov and Kurdubov (2012), Krasna et al. (2013) for VLBI; Yuan and Chao (2012) for GPS; Ray et al. (2005) for Altimetry.

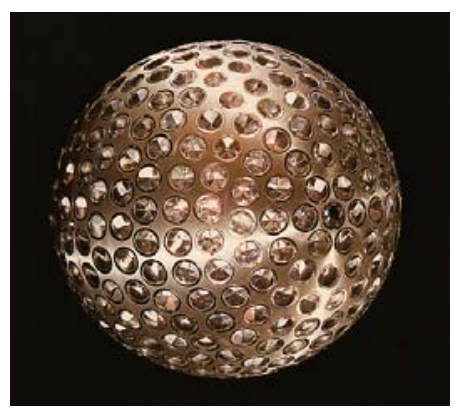

Figure 1a. The view of spherical satellites LAGEOS 1 and LAGEOS 2.

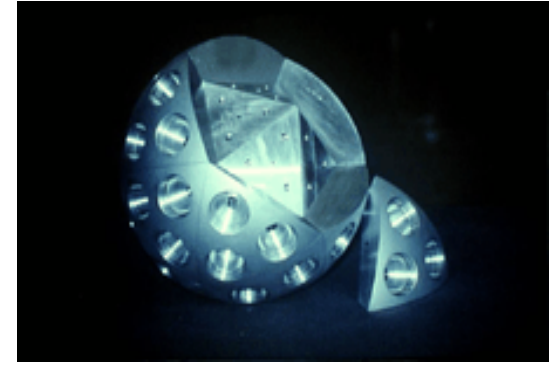

Figure 1b. The view of spherical satellites STARLETTE and STELLA.

All satellites are spherical and have an inclination equals to: $109.84^{\circ}$ (LAGEOS 1), $52.64^{\circ}$ (LAGEOS 2), $49.8^{\circ}$ (STARLETTE), $98.6^{\circ}$ (STELLA). The satellites are depicted in Figures $1 \mathrm{a}$ and $1 \mathrm{~b}$.

The satellite laser measurements for above described satellites were taken from the world database Crustal Dynamics Data Information System (CDDIS) and the EUROLAS Data Center (EDC). We analysed SLR data collected over a period of 2.5 years, from January 1, 2005 to July 1 2007. The distribution of the normal points per arc for LAGEOS 1 and LAGEOS 2 can be seen in Figure 2, for STARLETTE and STELLA in Figure 3. The number of normal points for LAGEOS 1, LAGEOS 2 and STARLETTE per arc is approximately the same, for satellite STELLA mean value of number of normal points is significantly smaller.

The raw SLR data for all satellites have been compressed from the SLR stations in to normal points based on 2 min intervals for LAGEOS 1 and LAGEOS 2 and on $15 \mathrm{sec}$ intervals for STARLETTE and STELLA. Because of atmospheric refraction, all normal points whose elevation was lower than 15 degrees were rejected in the solution. Description of normal points estimation in detail is given in Torrence et al. (1984) and Smith et al. (1991). 


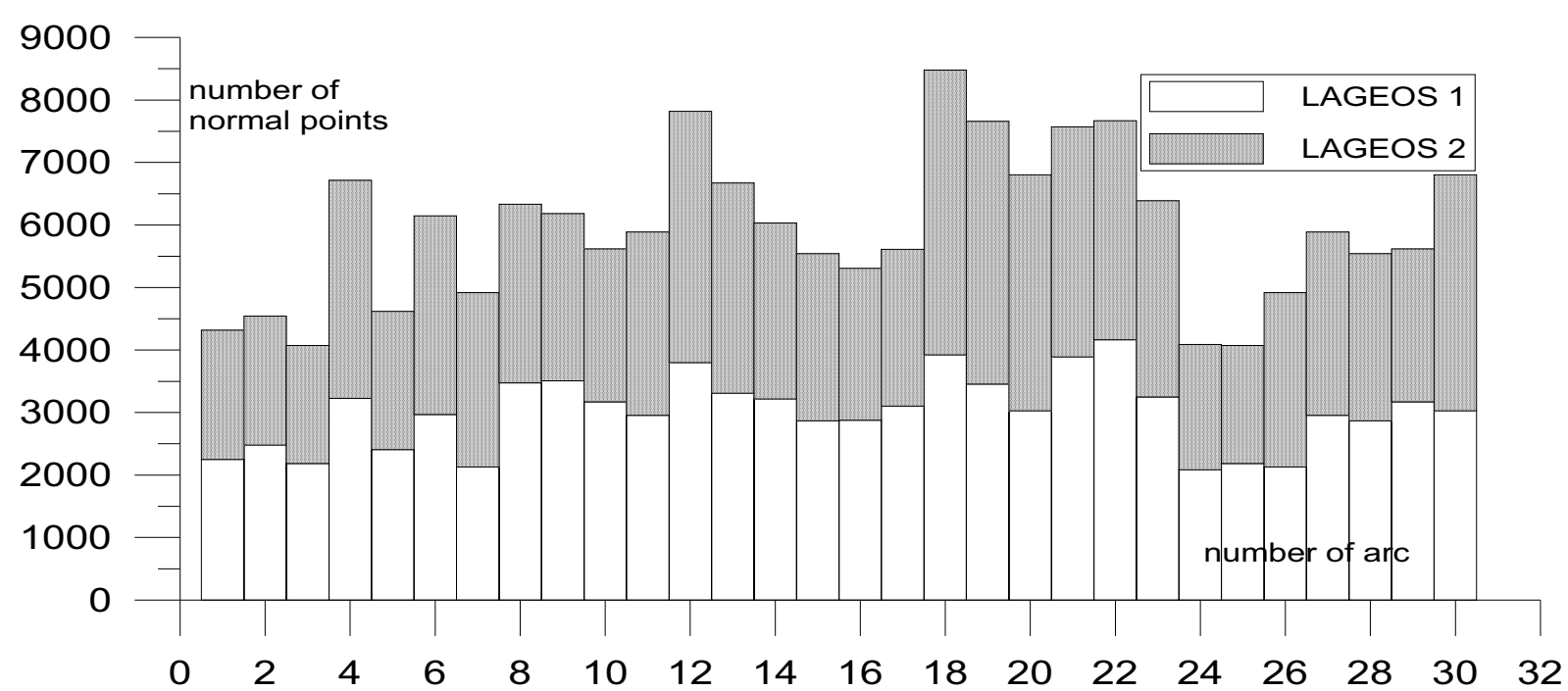

Figure 2. The number of normal points for LAGEOS 1 and LAGEOS 2 used in the solution. The database of normal points was processed in 30-day batches; in total 30 orbital arcs per satellite were used in analyses beginning from 01.01.2005. The horizontal axis gives the numbers of the orbital arcs.

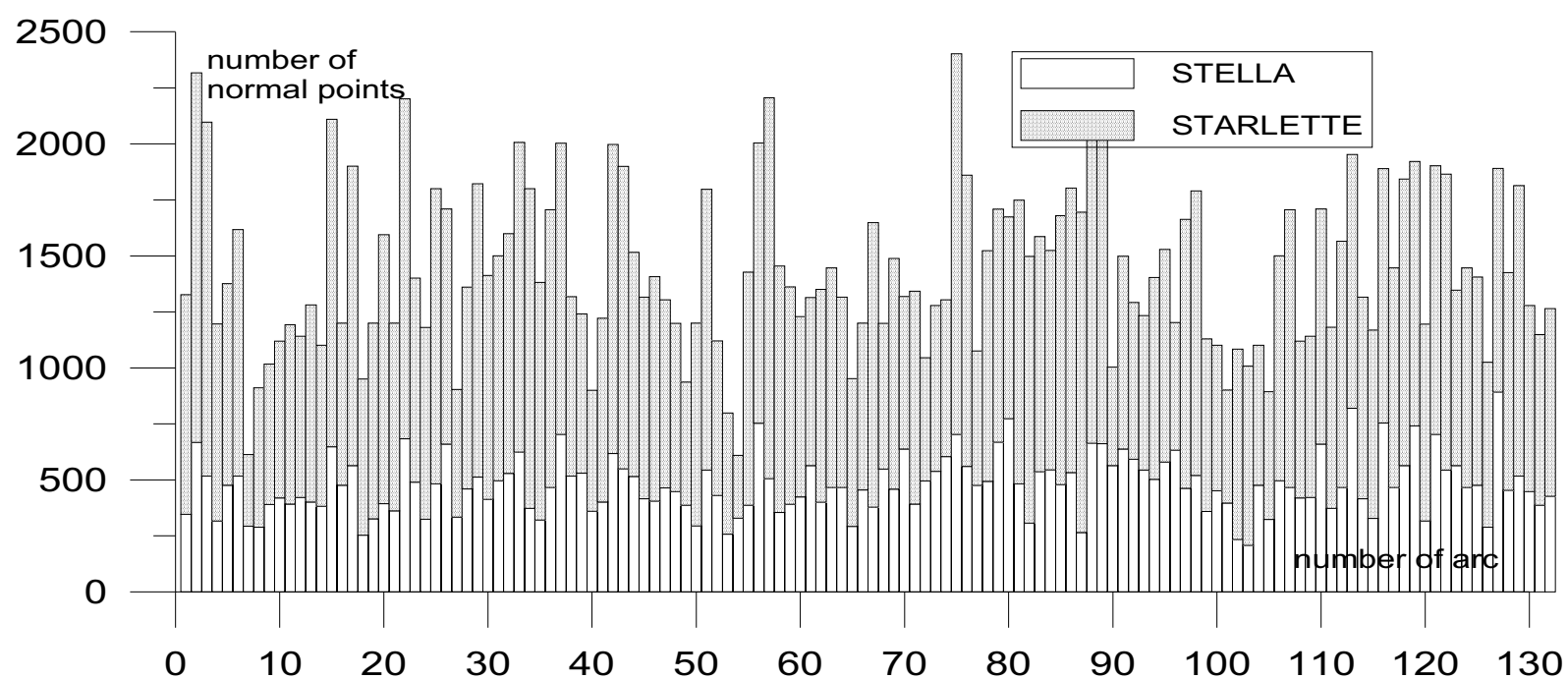

Figure 3. The number of normal points for STARLETTE and STELLA used in the solution.

The database of normal points was processed in 7-day batches; in total 132 orbital arcs per satellite were used in analyses beginning from 01.01.2005. The horizontal axis gives the numbers of the orbital arcs used in solution.

The database of normal points was processed in 30-day batches for LAGEOS 1 and LAGEOS 2 and 7-day batches for STARLETTE and STELLA (because the orbits of lowflying satellites - STARLETTE and STELLA are strongly influenced by perturbing forces, such as the irregularities of the gravity field, atmospheric drag, solid earth tides and ocean tides).

A subset of the satellite laser stations providing observations were selected; these 18 stations have known high quality data and their coordinates have been determined at the $1 \mathrm{~mm}$ level for each cartesian component in ITRF2008 (Altamimi et al. 2011). These coordinates have been determined from SLR measurements conducted continuously for many years. The Grasse and Shanghai stations are included in the solution in spite of the short time interval of their measurements (Grasse-until May 7, 2005, Shanghai-until April 14, 2005), the 
first in view of the high accuracy of normal points, the second because of its localization which improves configuration uniformity. The stations Yarragadee and Hartebeesthoek are very important in the solution because they are located in the Southern Hemisphere and improve the network configuration uniformity.

The weights for the stations were computed from the formula $W=1 / m_{s t}^{2}$, where $m_{s t}$ is the measurement accuracy for each station separately. In our solution laser range standard deviations of normal points taken from CDDIS file of data were adopted as $m_{S t}$. The mean values in units of two way time with a 1 picosecond granularity from 45 to 150 were obtained for the stations; they were computed for each arc independently. Generally, the data weight values for the observations are rather small, it can be due to an optimistic accuracy of our solution. Standard deviations for two way time intervals for each station are presented in Table 1.

Table.1 Standard deviations for two way time intervals for each station.

\begin{tabular}{|l|l|}
\hline \multicolumn{1}{|c|}{ Site name } & $\begin{array}{l}\text { Standard deviations for two } \\
\text { way time intervals (ps) }\end{array}$ \\
\hline Riga & 100 \\
\hline Fort Davis & 94 \\
\hline Yarragadee & 61 \\
\hline Potsdam & 124 \\
\hline Simosato & 138 \\
\hline Graz & 57 \\
\hline Greenbelt & 55 \\
\hline Monument Peak & 73 \\
\hline Shanghai & 149 \\
\hline Hartebeesthoek & 63 \\
\hline Borowiec & 116 \\
\hline Grasse & 98 \\
\hline San Fernando & 110 \\
\hline Herstmonceux & 94 \\
\hline Changchun & 112 \\
\hline Mount Stromlo & 53 \\
\hline Matera & 48 \\
\hline Wettzell & 118 \\
\hline
\end{tabular}

\section{DESCRIPTION OF PERFORMED ANALYSIS}

The solution was computed employing the software GEODYN II NASA/GSFC (McCarthy et al. 1993). The satellite orbits are computed using an 11th order predictor-corrector Cowell's method for the numerical integration of the satellite equations of motion in rectangular coordinates (Maury and Brodsky 1969). A step size of 100 seconds was used for satellites LAGEOS 1 and LAGEOS 2, and 15 seconds for STARLETTE and STELLA. The forces that perturb the satellite orbit needed to be modelled as accurately as possible.

The force model used in GEODYN II takes into account the following perturbations in the satellite motion, in line with IERS Conventions 2010 (Petit and Luzum 2010): 
- inhomogeneity of the Earth's gravity field,

- the gravity fields of the Sun and Moon,

- the gravity fields of the planets (Venus, Mars, Jupiter, Saturn),

- changes of the Earth gravity field induced by polar motion,

- changes in the Earth gravity field as a result of the ocean tide,

- changes in the Earth gravity field as a result of the Earth tide,

- direct solar radiation pressure coefficient $C_{R}$ estimated for each arc,

- atmospheric drag coefficient $C_{D}$ and MSIS 86 model (Hedin 1997), solved five values per week for STARLETTE and STELLA only (in our solution we analyzed two cases. In the first case we estimate five values per week for $C_{D}$ and in the second case seven values per week. Adding two unknowns to determine for each arc will increase by 240 unknowns in the whole adjustment. Greater number of unknowns caused a decrease in the value of $(O-C)$, but also increasing the value of the error estimated unknowns. Therefore, estimated of the five values per week for $C_{D}$ appears to be optimal),

- Earth albedo,

- Yarkowsky thermal drag,

- relativistic effects.

The gravitational potential of the Earth given by expression (1) changes with satellite altitude. For low satellites as STARLETTE and STELLA 80x80 spherical harmonics are used when modeling the orbit with an accuracy of 1-2 cm, but for high satellites such as LAGEOS 1 and LAGEOS 2 we use the spherical harmonic expansion 20x20 in line with Zelensky et al. (2008). The CSR gravity field model TEG4 (Tapley et al. 2002) was adopted. The perturbations caused by the third bodies - Moon, Sun and the planets Venus, Mars, Jupiter, Saturn - on the satellite orbit are computed using planetary positions for each SLR observational epoch derived from the JPL ephemeris DE200. Empirical accelerations in along-track, cross-track and radial directions were adjusted.

The numerical values for the precession-nutation model IAU 2000 have been adopted to computations shown in IERS Conventions 2010 (Petit and Luzum 2010).

The pole tide, polar motion $\left(x_{p}, y_{p}\right)$ and UT1 \{EOP05C04(IAU2000A)\}, ocean loading deformation and atmospheric pressure loading deformation model (Pavlis et al. 1998) were used in the solution. The center-of-mass correction equal to $25.1 \mathrm{~cm}$ for LAGEOS and equal to $7.5 \mathrm{~cm}$ for STARLETTE and STELLA were added to the laser ranging data. The values of the center of mass corrections are taken from CDDIS data base for analysed satellites and stations. For our study, nominal values $h_{2}=0.6078, l_{2}=0.0847$ IERS Conventions 2010 (Petit and Luzum 2010) were adopted as preliminary.

The study were performed in two parts, an overview of the parts is summarized in the block diagram shown in Figure 4. 
Pre-processing analysis of normal points taken from

CDDIS and EUROLAS for:

LAGEOS 1, LAGEOS 2, STARLETTE, STELLA

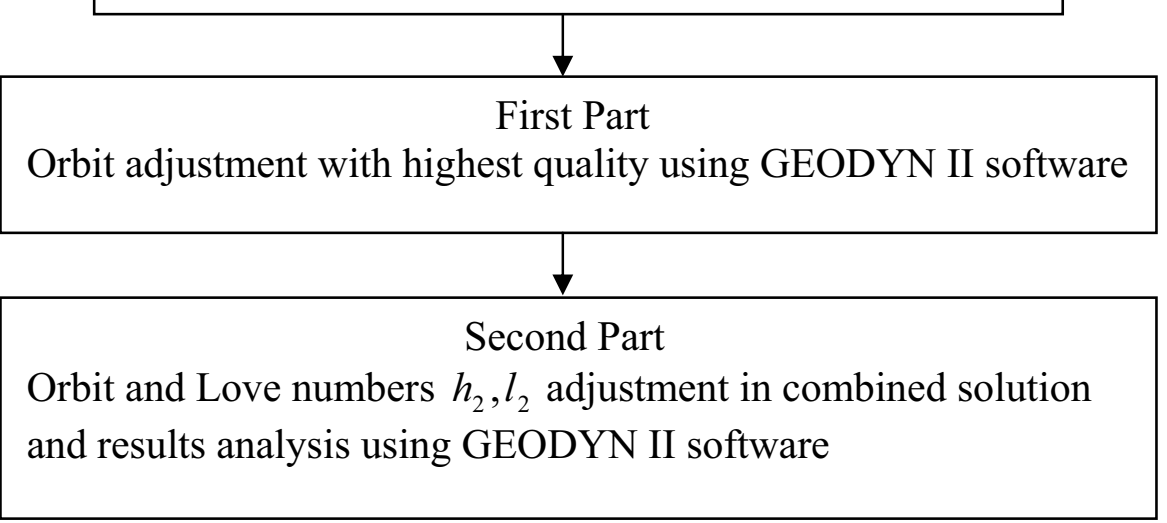

Figure 4. Diagram of the adjustment of the global elastic parameters $h_{2}, l_{2}$.

In the first part, the following unknowns were adjusted. The state vectors determining the position and velocity of the satellite at initial epoch, the light pressure coefficients and accelerations in along-track, cross-track and radial directions were adjusted for each 30-day orbital arc for LAGEOS 1, LAGEOS 2 and 7-day arc for STARLETTE and STELLA. In this step the random and systematic components of residuals $(\mathrm{O}-\mathrm{C})$ were analyzed. The outlier normal points that have a residual that exceeds three times the RMS of fit were removed. The range biases for network were analyzed in a way similar to that shown in Rutkowska and Noomen (1998). Agreement of the force model fit for two successive arcs was investigated from overlap. In our solution for selected 12-hour overlaps for LAGEOS 1 orbit, differences of satellite positions on the level of a few millimeter in radial direction and a few centimeter in along track and cross track direction were computed and shown in Figure 6 and Figure 7. The differences between satellite positions estimated from two successive arcs are directly proportional to the length of overlap. Because of limited size of the paper we selected some results which represent the entire solution appropriately.

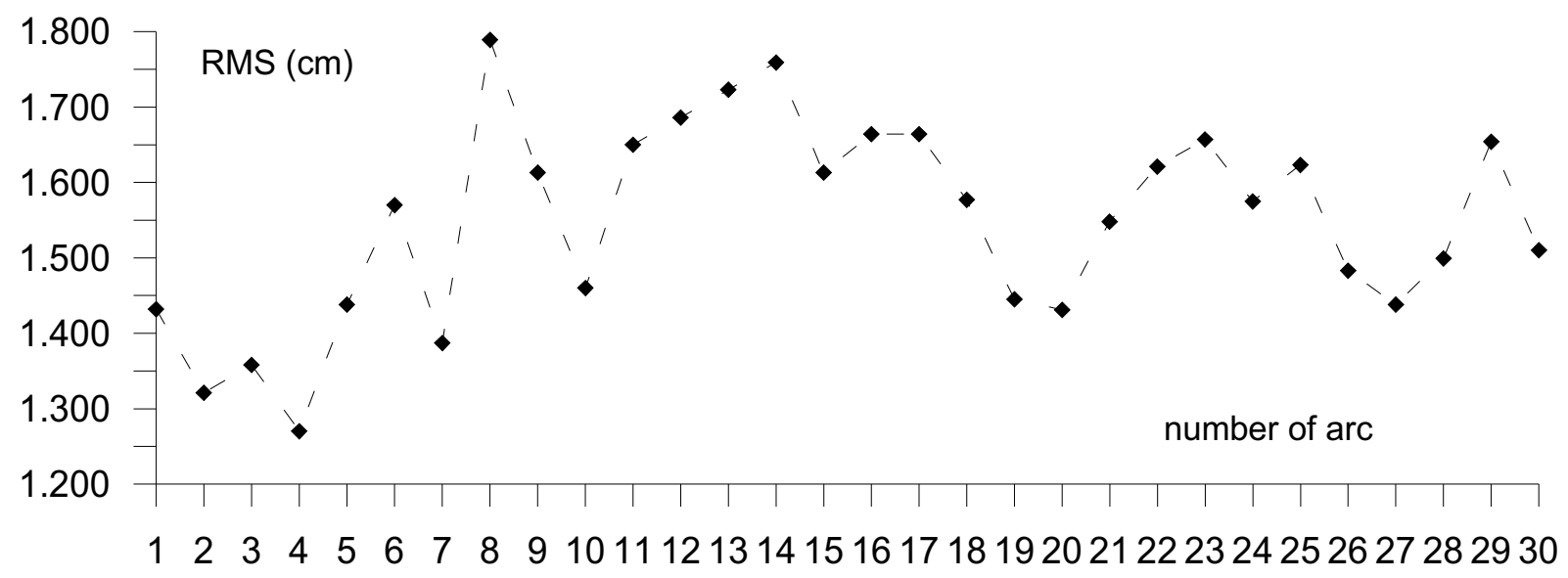

Figure 5. The RMS of the post-fit residuals for satellite LAGEOS 1 (in the second part). 


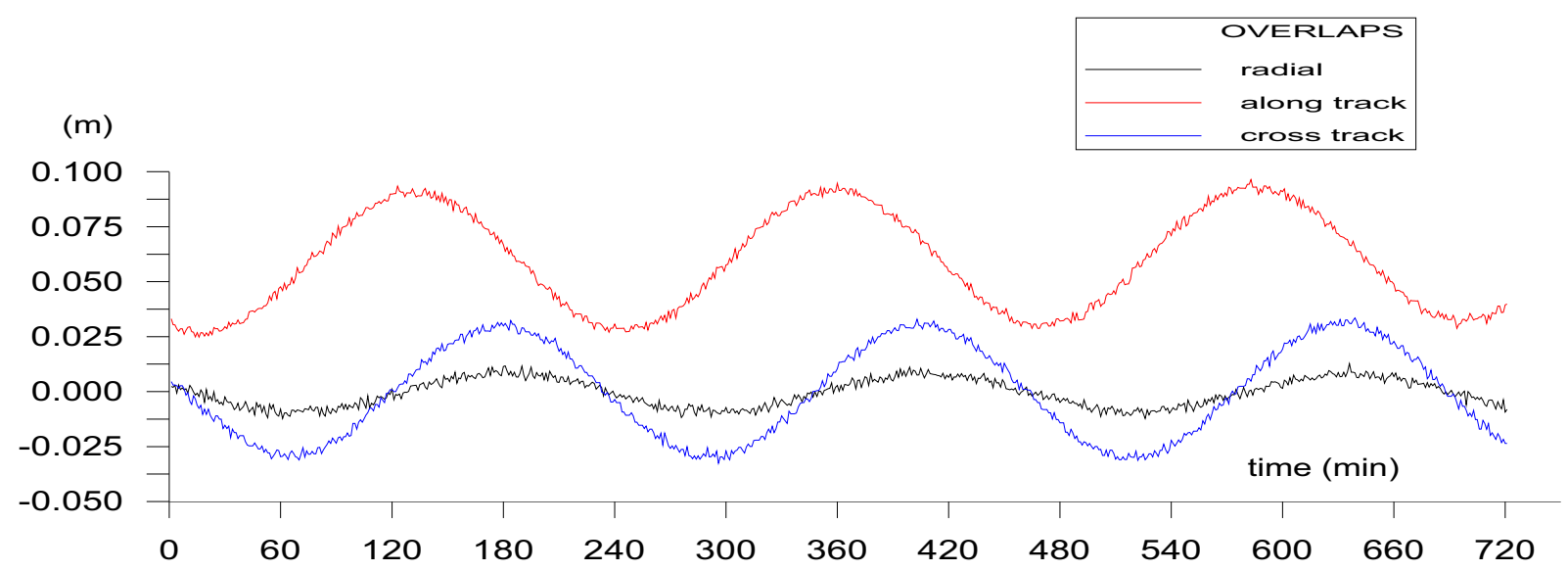

Figure 6. Overlaps (12-hour) between two orbits for LAGEOS 1 for day (19.05.2005).

Differences of satellite positions on the level of a few millimeters in radial direction and a few centimeters in along track and cross track direction were computed.

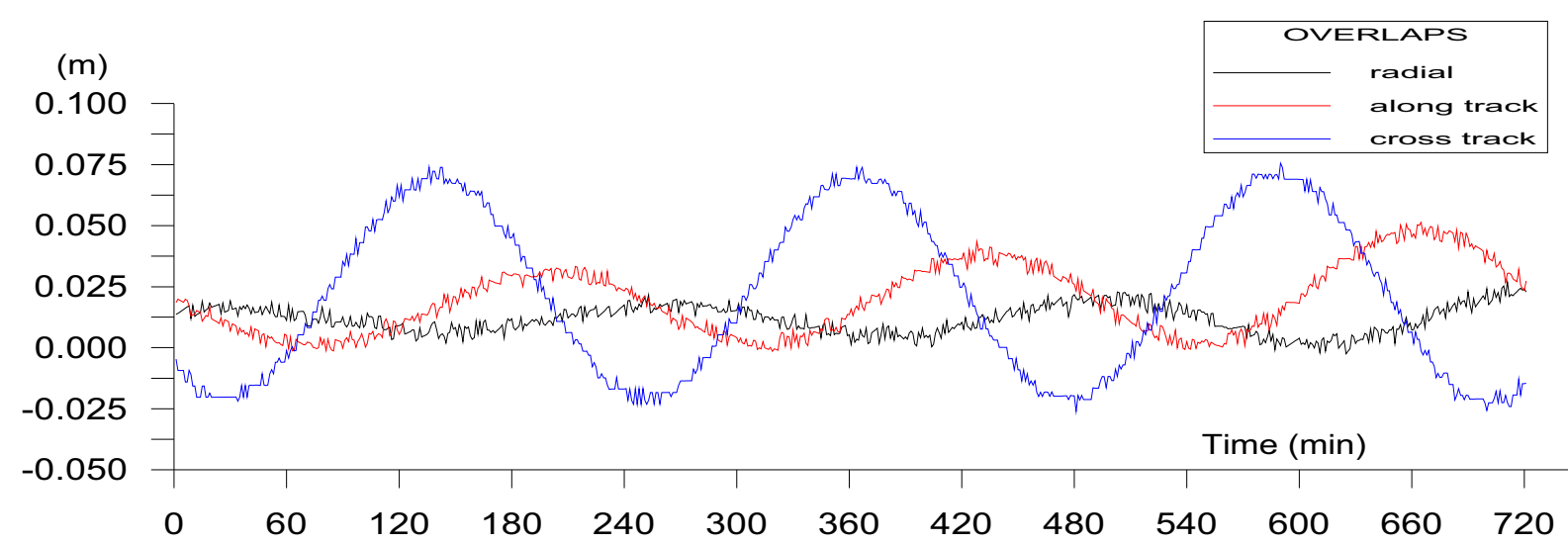

Figure 7. Overlaps (12-hour) between two orbits for LAGEOS 1, for day (13.08.2006). Differences of satellite positions on the level of a few millimeters in radial direction and a few centimeters in along track and cross track direction were computed.

The adjusted light pressure coefficients separately for each arc are at the level of 1.125-1.141 for LAGEOS 1 and LAGEOS 2.

The adjustment was performed in an iterative process with convergence criterion $\{\operatorname{RMS}(k)-\operatorname{RMS}(k-1)\}<0.01 \mathrm{~cm}$, where $(k)$ is the number of the iteration.

In our solution Keplerian elements for the initial epoch are converted into cartesian components (initial state vector). Satellites orbits are calculated by numerical integration of six equations: $\frac{d x}{d t}, \frac{d y}{d t}, \frac{d z}{d t}, \frac{d^{2} x}{d t^{2}}, \frac{d^{2} y}{d t^{2}}, \frac{d^{2} z}{d t^{2}}$. This solution allows the RMS of the post-fit residuals and RMS of satellite position at the initial epoch of arc to be estimated, as shown in Figure 5 for LAGEOS 1 and in Figure 8 for STELLA.

The RMS of the post-fit residuals and RMS of satellite position are computed from the following expressions;

RMS of the post-fit residuals $=\sqrt{\frac{\sum_{i=1}^{n}\left(O_{i}-C_{i}\right)^{2}}{n-1}}$, RMS of satellite position $=\sqrt{m_{X}^{2}+m_{Y}^{2}+m_{Z}^{2}}$ where: 
$i=1,2 \ldots . . n \quad$ - successive number of normal point,

$m_{X}, m_{Y}, m_{Z} \quad$ - errors of satellite positions in Cartesian coordinate system estimated from diagonal elements of covariance matrix.

Values of the post-fit residuals RMS calculated in the second part (see Figure 4) are 1-3 mm less than values for the RMS calculated in the first part (see Figure 4).

These values for RMS of the post-fit residuals for LAGEOS 1 and LAGEOS 2 (in the second part) are at the level of $1.2-1.9 \mathrm{~cm}$, mean value is equal to $1.56 \mathrm{~cm}$ for LAGEOS 1 and $1.52 \mathrm{~cm}$ for LAGEOS 2. This is shown in Figure 5. Values of the RMS errors of satellite positions (in the second part) computed at initial epoch are at the level of 1.1-2.5 cm for both satellites, the mean value is equal to $1.49 \mathrm{~cm}$ for LAGEOS 1 and $1.64 \mathrm{~cm}$ for LAGEOS 2 . Due to the limited size of this paper, results are only shown for one low satellite (STELLA) in Figures 8-11. The RMS errors of the post-fit residuals (in the second part) are at the level of $1.5-2.9 \mathrm{~cm}$, mean value is equal to $2.46 \mathrm{~cm}$. Similar order of the post-fit residuals RMS can be observed for STARLETTE.

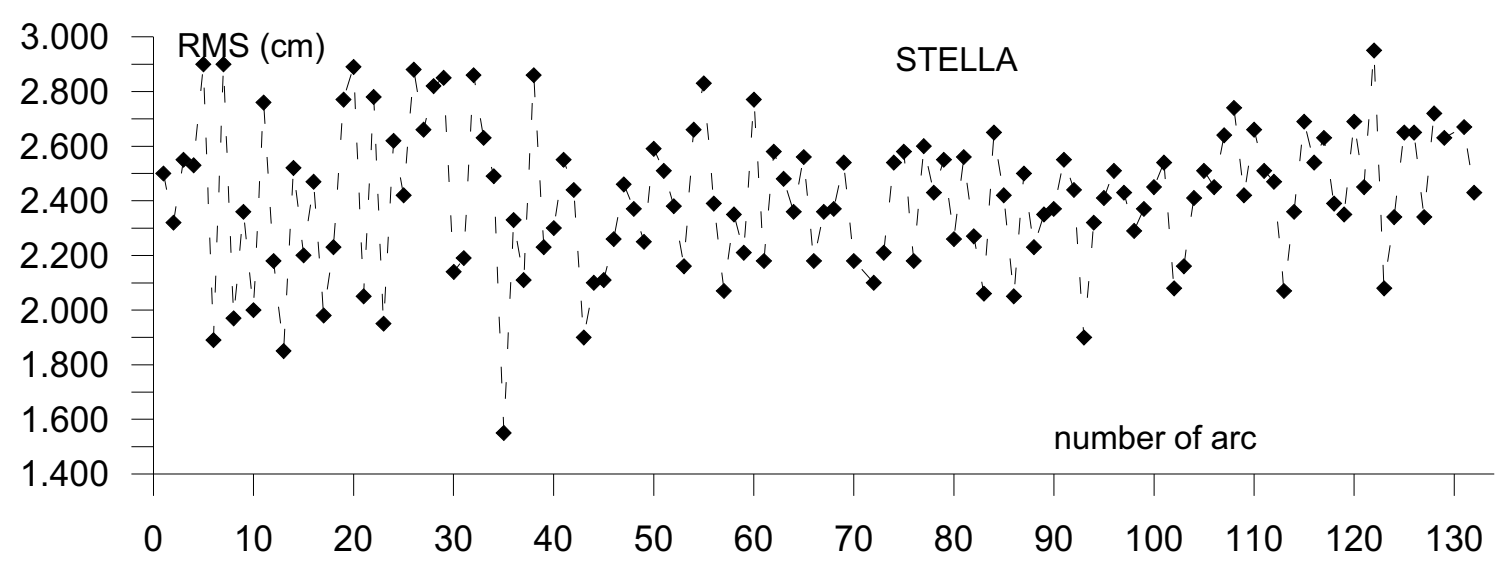

Figure 8. The RMS errors of the post-fit residuals for satellite STELLA (in the second part) are at the level of $1.5-2.9 \mathrm{~cm}$, mean value is equal to $2.46 \mathrm{~cm}$.

For the low satellites such as STELLA and STARLETTE it is necessary to estimate the effect of the perturbation caused by the resistance of the atmosphere (atmospheric drag) and the solar radiation pressure with high accuracy as the changes in the direct solar radiation pressure scaling coefficient $C_{R}$ are in the range of 1.1-1.3 (Figure 9) and the changes in the atmospheric drag coefficients $C_{D}$ are in the range 1.5-3 (Figures 10-11). These values scale the density model of the atmosphere and the solar radiation pressure in time.

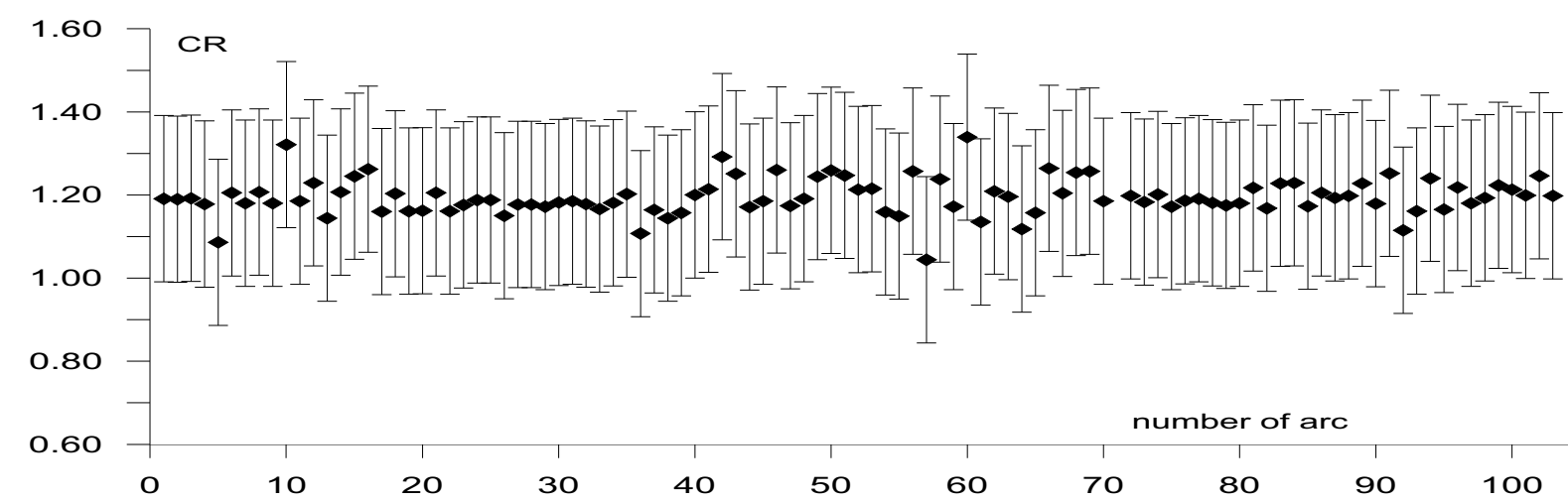

Figure 9. Solar radiation pressure coefficient computed for STELLA (one value for each arc in first part). 


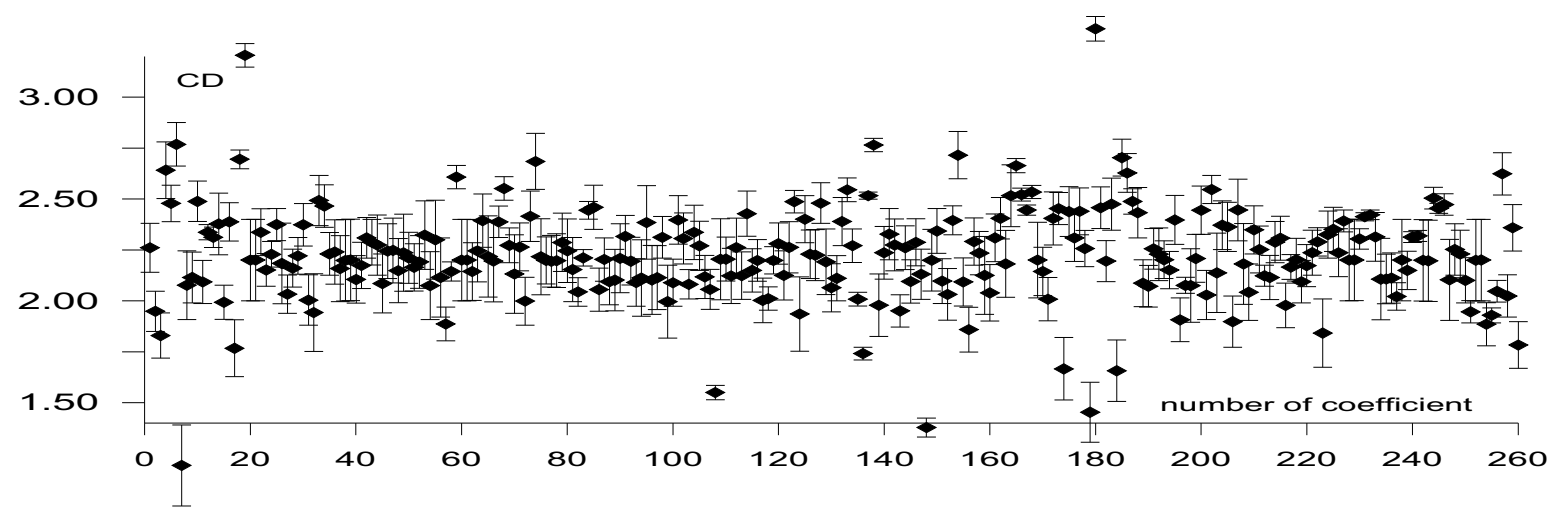

Figure 10. Atmospheric drag coefficient computed for STELLA (five values per week in first part).

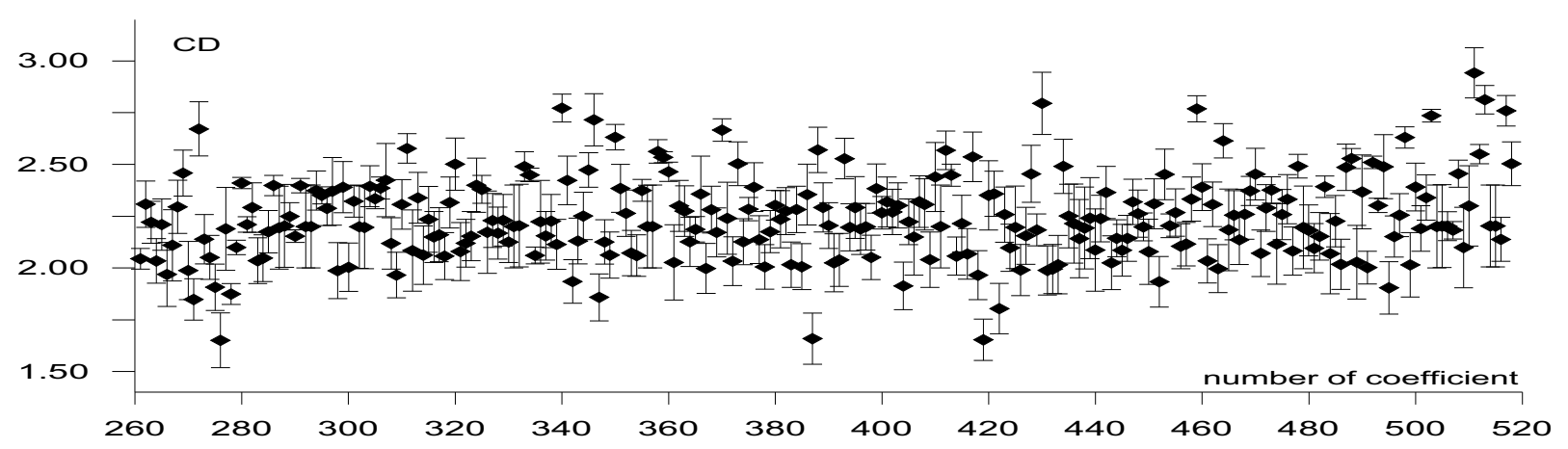

Figure 11. Atmospheric drag coefficient computed for STELLA (five values per week in first part).

After convergence of this first part, in the second part Love numbers are added and unknowns are determined in the combined solution for all the above described parameters.

In the second part, the sequential method (including a few steps) was adopted for our solution. In the first step, the elastic parameters were adjusted for two orbital arcs. In subsequent steps, arcs 3 and further were included one after the other using this sequential method. In each step, the parameters were adjusted once again, enabling the convergence and stability of two solutions to be observed, the first based on LAGEOS 1 and LAGEOS 2 data and the second on STARLETTE and STELLA data. The results of this analysis are shown in Figures 12-13 for $h_{2}$ and $l_{2}$, separately.

The set of two orbital arcs of the Love and Shida numbers differ significantly from their final values. Adding consecutive monthly (for LAGEOS1 and LAGEOS2) or weekly (for STELLA and STARLETTE) intervals allows to observe a slow convergence towards the final quantities.

The reason for this is that the stability of each solution and convergence for LAGEOS 1 and LAGEOS 2 has been attained after an interval of 2 years. This is shown in Figures 12-13 for LAGEOS 1 and LAGEOS 2 for common solution. In the common analysis of STARLETTE and STELLA data the estimated final Love numbers $h_{2}$ and $l_{2}$ are equal to $0.6132 \pm 0.0023$ and $0.0483 \pm 0.0100$ respectively. Estimation of the Shida number $l_{2}$ was unsuccessful for the low satellites. The discrepancies for Shida number between the high and 
low solutions were found to be on the level of $50 \%$ of the value, and on the level of fifty times the formal error.

The discrepancies between estimated Love numbers $h_{2}$ and $l_{2}$ in our solution and other independent solutions, derived by SLR, VLBI and altimetry as well as theoretical solutions are shown in Rutkowska and Jagoda (2010).

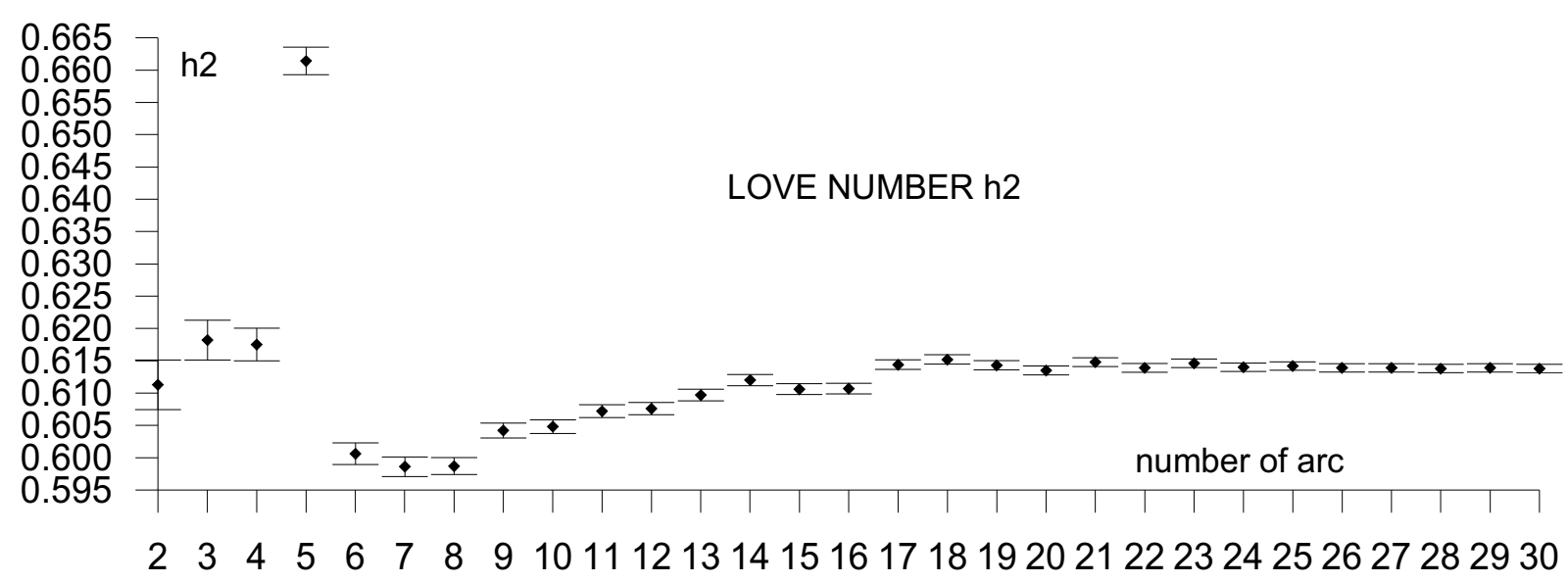

Figure 12. The sequential solution for the Love number $h_{2}$ based on data for LAGEOS 1 and LAGEOS 2 in the common adjustment. Stability of the estimated parameters $h_{2}$ and their errors becomes visible at about the 17-month time interval and does not change over the next twelve months. The final value of the Love number is equal to $0.6146 \pm 0.0006$.

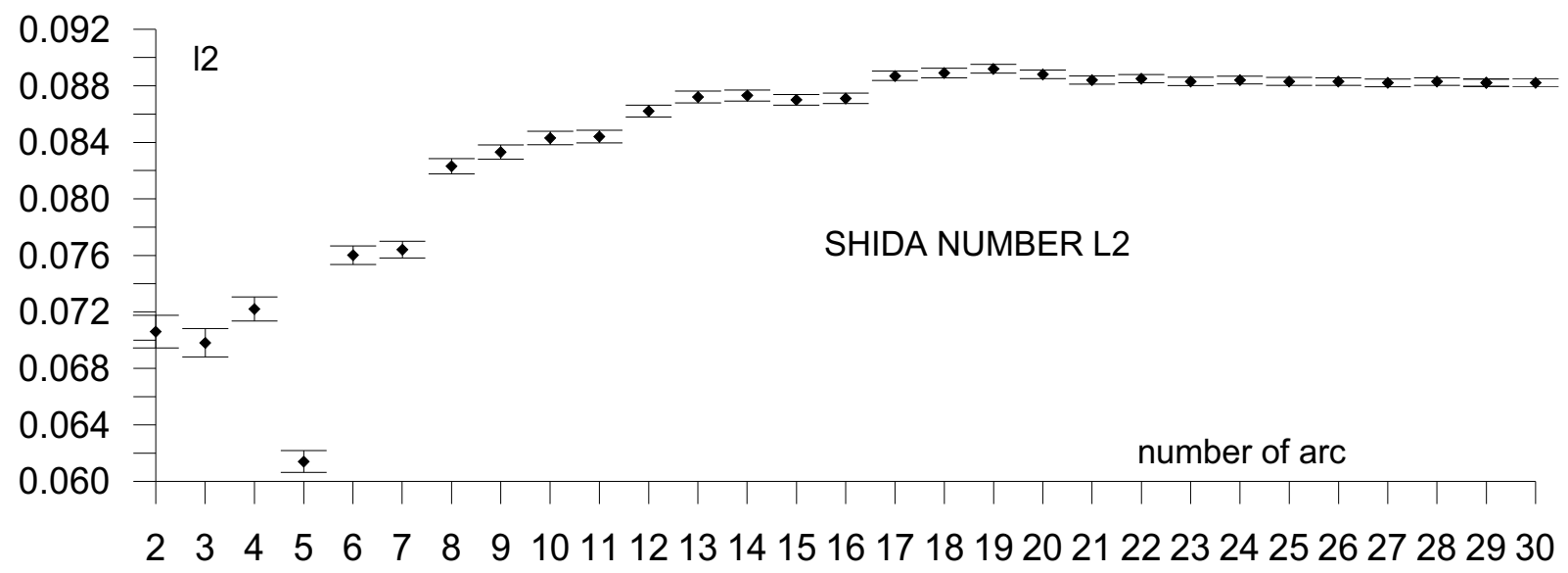

Figure 13. The sequential solution for the Shida number $l_{2}$ based on data for LAGEOS 1 and LAGEOS 2 in the common adjustment. Stability of the estimated parameters $l_{2}$ and their errors becomes visible for about 21-month time interval and does not change over the next nine months. The final value of the Shida number is equal to $0.0883 \pm 0.0003$. 


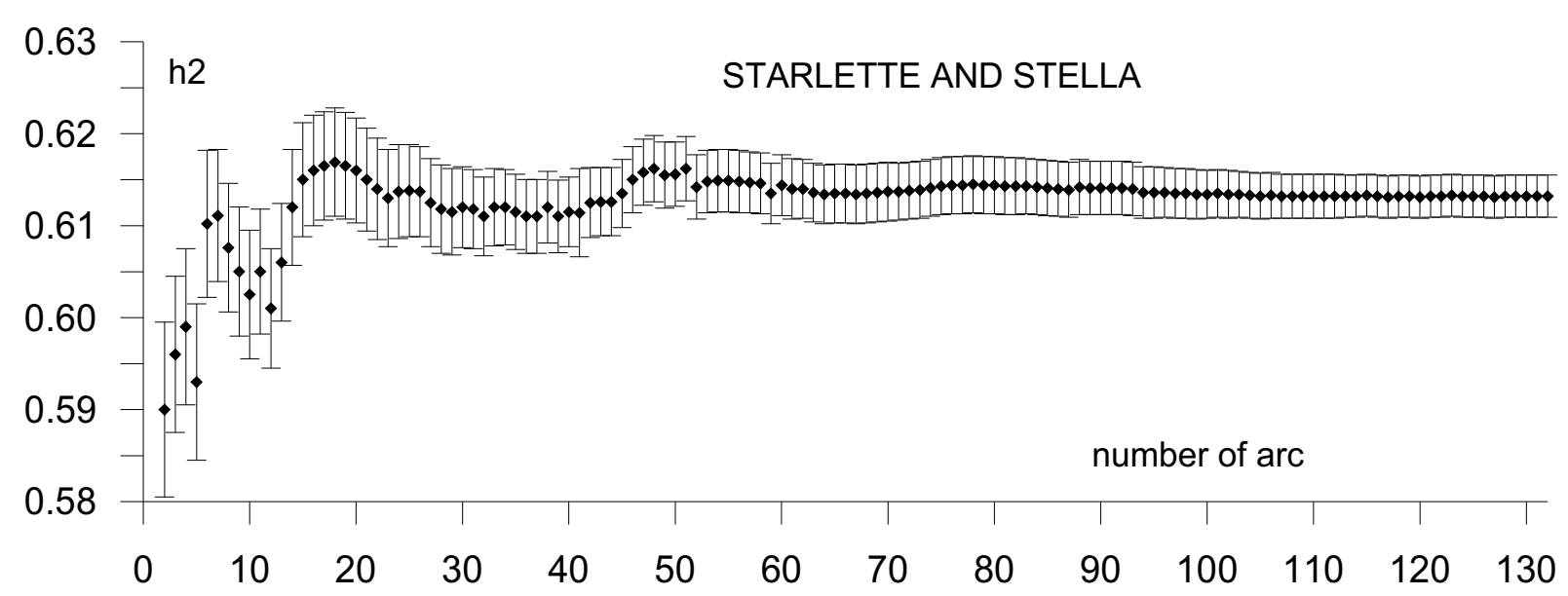

Figure 14. The sequential solution for the Love number $h_{2}$ based on data for STARLETTE and STELLA in the common adjustment. Stability of the estimated parameters $h_{2}$ and their errors becomes visible at about 14-month time interval and does not change over the next sixteen months. The final value of the Love number is equal to $0.6132 \pm 0.0023$.

\section{CONCLUSIONS}

We used SLR tracking data for high satellites LAGEOS 1, LAGEOS 2 and low satellites STARLETTE and STELLA to determine the elastic parameters $h_{2}$ and $l_{2}$ of the Earth in two separate solutions. Based on analysis of two solutions, we conclude that:

In the common analysis of LAGEOS 1 and LAGEOS 2 data the estimated final Love numbers $h_{2}$ and $l_{2}$ are equal to $0.6146 \pm 0.0006$ and $0.0883 \pm 0.0030$ respectively.

In the common analysis of STARLETTE and STELLA data the estimated final Love numbers $h_{2}$ and $l_{2}$ are equal to $0.6132 \pm 0.0023$ and $0.0483 \pm 0.0100$ respectively.

The discrepancy between the estimates in the common solution for high satellites LAGEOS 1 and LAGEOS 2 and in the common solution for low satellites STARLETTE and STELLA is equal to $0.23 \%$ for parameter $h_{2}$. Because this discrepancy is on the level of the formal error, in our evaluation the measurements for low satellites can be used in support of the solution obtained on the basis of high satellites. An exception is Shida number $l_{2}$, because the discrepancy of high and low solutions and with nominal value shown in Petit and Luzum (2010) are on the level of $50 \%$.

Based on data for LAGEOS 1 and LAGEOS 2, stability of the estimated elasticity Earth parameters $h_{2}$ and their errors becomes visible at about the 17-month time interval and does not change over the next twelve months. But based on data for STARLETTE and STELLA, stability of the estimated elasticity Earth parameters $h_{2}$ and their errors becomes visible at about 14-month time interval and does not change over the next sixteen months.

The error of estimated Love number based on data for STARLETTE and STELLA is about four times greater than the error estimated for LAGEOS 1 and LAGEOS 2 data. 


\section{REFERENCES}

Altamimi Z, Collilieux X, Métivier L (2011) ITRF2008: an improved solution of the international terrestrial reference frame. Journal of Geodesy, Volume 85, Issue 8

Diamante J, Williamson M (1972) Error Models for Solid Earth and Ocean Tidal Effects in Satellite Systems Analysis. Wolf Research and Development Corporation, Contract No. NAS 5-11735 Mod 57 for Goddard Space Flight Center, Greenbelt, Maryland, July 20, 1972

Eanes RJ, Schutz J, Tapley B (1983) Earth and Ocean Tide Effects on Lageos and Starlette, Proceedings of the Ninth International Symposium on Earth Tides, Kuo JT (ed.). E Sckweizerbart'sche Verlagabuchhandlung, 239-250, Stuttgart

Gubanov VS, Kurdubov SL (2012) Tidal deformations of the Earth from VLBI observations. Astronomy letters, Vol. 38, No.6

Hedin AE (1987) MSIS-86 Thermospheric Model. Journal of Geophysical Research 92

Jagoda M, Rutkowska M (2013) Estimation of the Love and Shida numbers: $h_{2}, l_{2}$ using SLR data for the low satellites. Advances In Space Research, Vol. 52

Krasna H, Bohm J, Schuh H (2013) Tidal Love and Shida numbers estimated by geodetic VLBI. Journal of Geodynamics 70 (2013) 21-27

Maury JL, Brodsky GD (1969) Cowell Type Numerical Integration as Applied to Satellite Orbit Computation., Goddard Space Flight Center X-553-69-46, December, 1969

McCarthy JJ, Rowton S, Moore D, Pavlis DE, Luthcke SB, Tsaoussi LS (1993) GEODYN II System Operation Manual, Vol.1-5, STX System Corp. Lanham MD 20706, USA

Petit G, Luzum B (2010) IERS Conventions (2010), IERS Technical Note No. 36, Verlag des Bundesamt für Kartographie und Geodäsie, Frankfurt an Main

Ray RD, Bettadpur S, Eanes RJ, Schrama EJO (1995) Geometrical determination of the Love number $h_{2}$ at four tidal frequencies. Geophysical Research Letters 22

Rutkowska M, Jagoda M (2010) Estimation of the elastic Earth parameters $\left(h_{2}, l_{2}\right)$ using SLR data. Advances In Space Research, Vol 46

Rutkowska M, Noomen R (1998) Range bias and station position analysis for network solution derived from SLR data. Proceedings $11^{\text {th }}$ International Workshop on laser ranging, Deggendorf, pp 70-79

Smith DE, Kolenkiewicz R, Dunn PJ, et al (1991) LAGEOS Geodetic Analysis SL7.1, NASA, Technical Memorandum 104549

Tapley BD, Chambers DP, Cheng MK, et al (2002) The TEG-4 Earth Gravity Field Model. Paper presented at the XXV General Assembly of the European Geophysical Society, Nice, 27 April, 2002

Torrence MH, Klosko SM, Christodoulidis DC (1984) The Construction and Testing of Normal Points at Goddard Space Flight Center. 5th International Workshop on Laser ranging Instrumentation, Herstmonceux, UK, published by Geodetic Institute, Univ. Bonn, 506- 511 
Yuan L, Chao BF (2012) Analysis of tidal signals in surface displacement measuredy by a dense continuous GPS array. Earth and Planetary Science Letters 355-356 (2012) 255-261

Zelensky NR, Lemoine FG, Rowlands DD, et al (2008) Sub-centimeter SLR precision with the SLR2005/LPOD2005 network. Proceedings of the 16-th International Workshop on Laser Ranging, pp 215-223, Poznań, October, 2008

Received: 2015-01-26,

Reviewed: 2015-03-18, by L. Combrinck,

Accepted: 2015-09-14. 